\title{
Day-ahead forecasting approach for energy consumption of an office building using support vector machines
}

\author{
Aria Jozi ${ }^{1}$, Tiago Pinto ${ }^{2}$, Isabel Praça ${ }^{1}, Z_{\text {Zita Vale }}^{3}$ \\ ${ }^{1}$ GECAD research group, Polytechnic of Porto (ISEP/IPP) \\ Porto, Portugal \\ \{arjoz, icp\}@isep.ipp.pt \\ ${ }^{2}$ BISITE research group, University of Salamanca \\ Salamanca, Spain \\ tpinto@usal.es \\ ${ }^{3}$ Polytechnic of Porto (ISEP/IPP) \\ zav@isep.ipp.pt
}

\begin{abstract}
This paper presents a Support Vector Machine (SVM) based approach for energy consumption forecasting. The proposed approach includes the combination of both the historic log of past consumption data and the history of contextual information. By combining variables that influence the electrical energy consumption, such as the temperature, luminosity, seasonality, with the log of consumption data, it is possible for the proposed method by find patterns and correlations between the different sources of data and therefore improves the forecasting performance. A case study based on real data from a pilot microgrid located at the GECAD campus in the Polytechnic of Porto is presented. Data from the pilot buildings are used, and the results are compared to those achieved by several states of the art forecasting approaches. Results show that the proposed method can reach lower forecasting errors than the other considered methods.
\end{abstract}

Keywords: Data series analysis, Energy consumption forecasting, Support vector machines; office building

\section{INTRODUCTION}

Worldwide policies are placing the consumer as the central piece of future power and energy systems [1]. Consumption flexibility is seen as the most promising solution to enable the system to cope with the increasing penetration of renewable energy sources. While the massive investment in energy efficiency measures contributes to lowering the overall energy consumption, thus being crucial to pave the path towards the dream of a fully renewable energy based energy system; the uncertainty and variability of renewable based generation bring additional challenges that need to be addressed [2]. Being able to take advantage from the consumers' flexibility potential is, in this way, essential to enable guaranteeing the balance between (variable) generation and consumption[3].

This work has received funding from the European Union's Horizon 2020 research and innovation programme under the Marie Sklodowska-Curie grant agreement No 641794 (project DREAM-GO) and from FEDER Funds through COMPETE program and from National Funds through FCT under the project UID/EEA/00760/2013
Adequate consumption forecasting methods become, therefore, urgent in this domain. Although consumption estimation has always been necessary for power and energy systems, so that the dispatchable generation could be controlled to meet the demand; nowadays the need for efficient and effective approaches arises [4]. New methods that are able to deal with the large number of variables with influence on energy consumption (e.g., temperature, luminosity, seasonality, among many others), and take the most advantage out of the correlation between these factors, are needed. Moreover, the response time of these new models is required to be as quick as possible, so that fast responses to generation and prices fluctuations can be provided, and the most potential from consumption flexibility can be explored [5].

Energy consumption forecasting has been traditionally tackled by a variety of different methods, in particular, the socalled conventional methods (mostly based on regression models), and artificial intelligence approaches, as discussed in the review presented in [6]. Among the most widely used traditional models are the regression approaches based, e.g., on ARIMA or GARCH [7]. Several works have also taken advantage of the advantages from regression based forecasting to model probabilistic prediction approaches, e.g. [8]. On the artificial intelligence side, a meaningful review of intelligent approaches for load forecasting is presented in [9]. The most widely used approach in this domain is the Artificial Neural Networks (ANN), e.g., the work presented in [10]. Several applications of fuzzy logic have also been proposed, and are achieving promising results [11]. Moreover, combinations between ANN and fuzzy methods, called neuro-fuzzy inference systems, are arising as prominent methods in this domain [12]. However, multiple works involving Support Vector Machines (SVM), are surpassing the results achieved from traditional regression methods and ANN based approaches, as shown in [13]. In fact, when combined with other methods, such as metaheuristic optimization methods, SVMs are proving to be 
powerful tools for forecasting in multiple domains, and, in particular, for consumption forecasting [14].

Although there have been significant advances in consumption forecasting in recent years; there are still several gaps that need to be addressed in order to enable reaching better forecasting results in faster execution times. The capability of considering contextual data and combining it with the historical consumption data in order to find patterns among these variables is still under-developed. Also, the efficiency in data processing so that predictions can be achieved in due time, still needs further improvement.

This paper contributes to overcoming these gaps by presenting an SVM based approach for consumption forecasting, combining contextual information with the historic log of consumption. The proposed approach includes the access to real data from a pilot microgrid, composed by several buildings at the campus of the School of Engineering of the Polytechnic of Porto. The consumption is forecasted using the proposed approach and validated against the real consumption data measured in the pilot buildings. The performance of the proposed approach is also compared to that of several states of the art forecasting approaches, namely hybrid fuzzy inference systems (HyFIS) [15], Wang and Mendel's Fuzzy Rule Learning Method (WM) [16] and a genetic fuzzy system for fuzzy rule learning based on the MOGUL methodology (GFS.FR.MOGUL) [17]. Results show that the proposed methodology is able to reach lower forecasting errors than the considered state of the art forecasting methods, by using the contextual data as the input of the forecasting process.

After this introductory section, section II presents the proposed methodology, and also the data used in the scope of this work. Section III presents a case study based on real data and includes a discussion on the achieved results. Finally, section IV provides the most relevant conclusions of this work.

\section{MATERIAL AND METHODS}

This work proposes a day-ahead energy consumption forecasting approach using SVM as the primary forecasting method. More thee Fuzzy Rule Based Systems(FRBS) are also used to compare the results and find the best forecasting method which presents the most trustable performance. Namely as, the Hybrid Neural Fuzzy Interface System (HyFIS)[15], Wang and Mendel's Fuzzy Rule Learning Method (WM)[16] and a genetic fuzzy system for fuzzy rule learning based on the MOGUL methodology (GFS.FR.MOGUL) [17]. This study uses the real energy consumption data form building $\mathrm{N}$ of the Research Group on Intelligent Engineering and Computing for Advanced Innovation and Development (GECAD) research center located in ISEP/IPP, Porto, Portugal. These data are collected and stored through SOICAM (SCADA Office Intelligent Context Awareness Management) [18], a system that is used to manage and simulate the GECAD campus microgrid. All of the forecasting methods are implemented in the $\mathrm{R}$ programming language. The detailed implementation of the process and the result are included in the following section.

\section{A. Support Vector Machines}

Support Vector Machines (SVM's) are a field of supervised machine learning methods and are one of the most known methods in the area of forecasting. The first running kernel of SVM was created in the sequence of by Vapnik [19], implementing a generalization of the nonlinear algorithm Generalized Portrait and only for classification and linear problems. Vapnik developed the statistical learning theory further in 1979. Finally, the current form of the SVM approach was presented in 1992, with a paper at the COLT conference [20]. The information to use in an SVM must follow the format suggested in equation (1):

$$
\left(y_{1}, x_{1}\right), \ldots,\left(y_{i}, x_{i}\right), x \in R^{n}, y \in R
$$

Where each example $\mathrm{x}_{i}$ is a space vector example; $\mathrm{y}_{i}$ has a corresponding value; $\mathrm{n}$ is the size of training data. For classification: $\mathrm{y}_{i}$ assumes finite values; in binary classifications: $\mathrm{y}_{i} \in\{+1,-1\}$; in digit recognition: $\mathrm{y}_{i} \in\{1,2,3,4,5,6,7,8,9,0\}$; and for regression purposes, $\mathrm{y}_{i}$ is a real number $\left(\mathrm{y}_{i} \in \mathrm{R}\right)$.

The implementation of SVM requires considering some critical aspects, namely:

- Feature Space

- Loss Functions

- Kernel Functions

The most applicable kernels for time series forecasting, as in the problem considered in this work, are the Radial Basis Function (RBF) and the exponential Radial Basis Function (eRBF). These two kernels are directed explicitly to regression in time series data. The SVM approach takes as parameters:

- $\quad$ training Limit - limit number of training data;

- $\quad$ kernel - kernel that is used in the regression process;

- $\varepsilon$-insensitive - the error that is permitted, i.e. the lower the value, the higher the exigency of the regression process;

- limit - limit of the kernel function;

- $\sigma$ - the angle of the kernel function;

- offset - offset of the kernel function.

A suitable combination of these parameters is essential to achieve quality results. The most suitable combination is highly dependable on the characteristics and particularities of each distinct problem; therefore, an exhaustive sensitivity analysis must be performed for each application, in order to achieve conclusions on the best combinations of parameters that should be used. SVM method in this work is implemented based on $\mathrm{R}$ programming language using the "e1071" package[21].

\section{B. Fuzzy Rule Based Systems (FRBS)}

Fuzzy rule-based systems (FRBSs) are a group of forecasting algorithms included in the "FRBS" package of $\mathrm{R}$ programming language [22]. These methods are based on the fuzzy concept proposed by Zadeh in 1965 [23] and can be divided into five groups based on the different used approaches in their implementation.

1. FRBS based on space partition

2. FRBS based on neural networks 
3. FRBS based on the clustering approach

4. FRBS based on genetic algorithms

5. FRBS based on the gradient descent method

For this work three forecasting method from this package have been used namely as Hybrid Neural Fuzzy Interface System (HyFIS), Wang and Mendel's Fuzzy Rule Learning Method (WM) and a genetic fuzzy system for fuzzy rule learning based on the MOGUL methodology (GFS.FR.MOGUL).

HyFIS is one of the FRBS based on neural networks methods and is the most known method of this package. This method is a combination of Fuzzy Rules and Neural Networks that includes two learning phases [24]:

- $\quad$ The first phase concerns the structure learning, i.e., finding the rules by using the knowledge acquisition module;

- The second phase regards the parameter learning phase for tuning fuzzy membership functions to achieve the desired level of performance [25].

The advantage of using HyFIS is that the fuzzy rule base can be easily updated when there is newly available data. When there is a new available pair data, a rule is created for this data, and this new rule updates the fuzzy rule base. A more detailed explanation of the implementation of this method can be found in[15].

The second chosen FRBS method for this work is WM proposed by Wang and Mendel in 1991 [16]. The process of fuzzy rule bases generation in this method includes four steps:

1. Divide the Input and Output Spaces into Fuzzy Regions.

2. Generate Fuzzy Rules from Given Data Pairs

3. Assign a degree to each rule

4. Create a Combined Fuzzy Rule Base

This method has been known because of having a simple structure with a good performance. In [16] has been presented a complete explanation of the steps of this method as well as the details of the implementation process.

GFS.FR.MOGUL is a forecasting method that implements a genetic algorithm determining the structure of the fuzzy IFTHEN rules and the membership function parameters. Two general types of fuzzy IF-THEN rules are considered:

- Descriptive rules.

- Approximate/free semantic approaches.

In the first type, the linguistic labels illustrate a real-world semantic, and the linguistic labels are uniformly defined for all rules. In contrast, in the approximate approach, there is any associated linguistic

label. The presented work in [17] includes a complete explanation about the structure of this method.

\section{Database}

The real energy consumption of the Building N of GECAD facilities located in Porto, Portugal has been chosen to be used in this work. This building includes five energy meters that each one stores the energy consumption of a specific part of the building by a time interval of 10 seconds. These energy meters store the energy consumption of the air conditioning system, lights, and electrical sockets separately. All the electrical information of this building is stored in the SQL server of the GECAD. This server includes several databases where various historical data related to the building are stored such as the solar radiation of the area of the building as well as the environmental temperature of the related place which will be used in this forecasting process.

\section{RESULTS AND DISCUSSION}

The objective of this study is to find a new forecasting approach for day-ahead energy consumption prediction which presents a more trustable performance comparing to the previous works. SVM will be used as the main forecasting method of this work, and also the results of this method will be compared to the results of three Fuzzy Rule Based forecasting methods namely as HyFIS, WM, and GFS.FR.MOGUL.

Two Training strategies are proposed in this study. In the first forecasting strategy, the total energy consumption is divided into the consumption of air conditioning systems, Lights and electrical sockets and the forecasting methods will predict the consumption of these three types of consumers separately. To predict the consumption of each type of consumers, the methods will be trained by the values of energy consumption of the intended consumer during the past 11 weeks. The output of the methods will present the energy consumption of the consumers in the next 24 hours, and the sum of these three values presents the total energy consumption of the building. This strategy has been presented in [17]. Where the GFS.FR. MOGUL presents the best performance. In this work, the SVM is proposed to be used by the same data set to forecast the energy consumption of the next 24 hours. Table 1 presents the forecasted total energy consumption by these four methods for 16/11/2016.

As it can be seen in table 1 the provided results by SVM are closer to the real values. To compare the results of these methods the Mean Absolute Percentage Error (MAPE) is used in this work. The average MAPE error of SVM when the first strategy has been used is $9.44 \%$, while this value for HyFIS is $18.84 \%$, for $\mathrm{WM}$ is $18.79 \%$ and for GFS.FR.MOGUL is $10.01 \%$. These results prove that SVM can estimate a more reliable energy consumption profile for the next 24 hours when the first training strategy is used.

The second strategy takes advantage of using a second variable in the phase of training. In this strategy as same as the first one the energy consumption is divided into the consumption of HVAC, Lights, and e electrical Sockets, and the methods will predict the consumption of these three consumers separately. Also, the consumption of the electrical Sockets will be forecasted in the same way which means that methods to predict this consumption will be trained by the value consumption of the electrical sockets during the past 11 weeks. On the other hand, two meteorological variables namely as environmental temperature and solar radiation will be used to train the methods to predict the consumption of the Lights and HVACs. In the case of lights, the methods receive the values of the energy consumption and solar radiation of past 11 weeks as the training data and forecast the amount of the consumption of the lights for the next 24 hours. Also, for HVAC the combination of the value 
of consumed energy by HVACs and environmental temperature will be used as the training data to predict the consumption of HVAC in the next 24 hours. Figure 1 presents the structure of this forecasting process.

Table 1 - Forecasted consumption values based on the first strategy

Hour Real SVM HyFIS WM GFS.FR.MOGUL

\begin{tabular}{|c|c|c|c|c|c|}
\hline $0: 00$ & 1502 & 1594 & 1688 & 1682 & 1683 \\
\hline $1: 00$ & 1602 & 1578 & 1350 & 1350 & 1785 \\
\hline $2: 00$ & 1462 & 1536 & 1319 & 1323 & 1728 \\
\hline $3: 00$ & 1499 & 1708 & 1900 & 1887 & 1752 \\
\hline $4: 00$ & 1467 & 1575 & 1836 & 1836 & 1572 \\
\hline $5: 00$ & 1525 & 1535 & 1612 & 1609 & 1769 \\
\hline $6: 00$ & 2065 & 1552 & 1659 & 1660 & 1555 \\
\hline $7: 00$ & 1447 & 1527 & 1443 & 1430 & 1807 \\
\hline $8: 00$ & 1534 & 1531 & 1439 & 1439 & 1598 \\
\hline 9:00 & 2186 & 2109 & 1751 & 1821 & 2360 \\
\hline 10:00 & 2447 & 2327 & 1932 & 1957 & 2505 \\
\hline 11:00 & 2640 & 2925 & 2011 & 2011 & 2834 \\
\hline $12: 00$ & 3235 & 2735 & 1985 & 1873 & 3184 \\
\hline 13:00 & 3566 & 2980 & 2551 & 2551 & 3232 \\
\hline $14: 00$ & 3164 & 3508 & 2738 & 2712 & 3556 \\
\hline 15:00 & 3621 & 3446 & 3089 & 3089 & 3880 \\
\hline $16: 00$ & 3710 & 3482 & 1779 & 1779 & 3722 \\
\hline $17: 00$ & 3399 & 3174 & 2562 & 2562 & 3585 \\
\hline 18:00 & 3031 & 2545 & 2536 & 2521 & 2702 \\
\hline 19:00 & 2324 & 1883 & 2107 & 2110 & 2140 \\
\hline 20:00 & 1791 & 1705 & 2205 & 2205 & 1854 \\
\hline 21:00 & 2388 & 1673 & 1702 & 1705 & 2022 \\
\hline $22: 00$ & 1820 & 1618 & 1748 & 1762 & 1997 \\
\hline 23:00 & 1756 & 1770 & 1537 & 1538 & 1814 \\
\hline
\end{tabular}

To predict every consumption value, the forecasting methods receives a .csv file which includes the required data to train the methods to predict the target values. These files are created by a Java based application which is connected to the databases. This application collects and calculates the hourly consumptions and creates a .csv file for every type of consumers. These input files include three sets of data namely as Traininput, Trainoutput, and Test. The Traininput and Trainoutput tables are used to train the methods and create the forecasting model. The Test table is the main input of the process which the trained model receives to estimate the final value. The Test table includes the consumption value of the intended consumer in the same hour as the target hour from past 14 days.
The same day as the first strategy has been chosen as a target day to evaluate the performance of this forecasting strategy. In the case of Lights, the forecasting process only predicts the consumption from 9:00 to 20:00 and for the rest of the hours zero consumption is considered. Table 2 presents the predicted values by the forecasting methods using the second strategy for 24 hours of 16/11/2016.

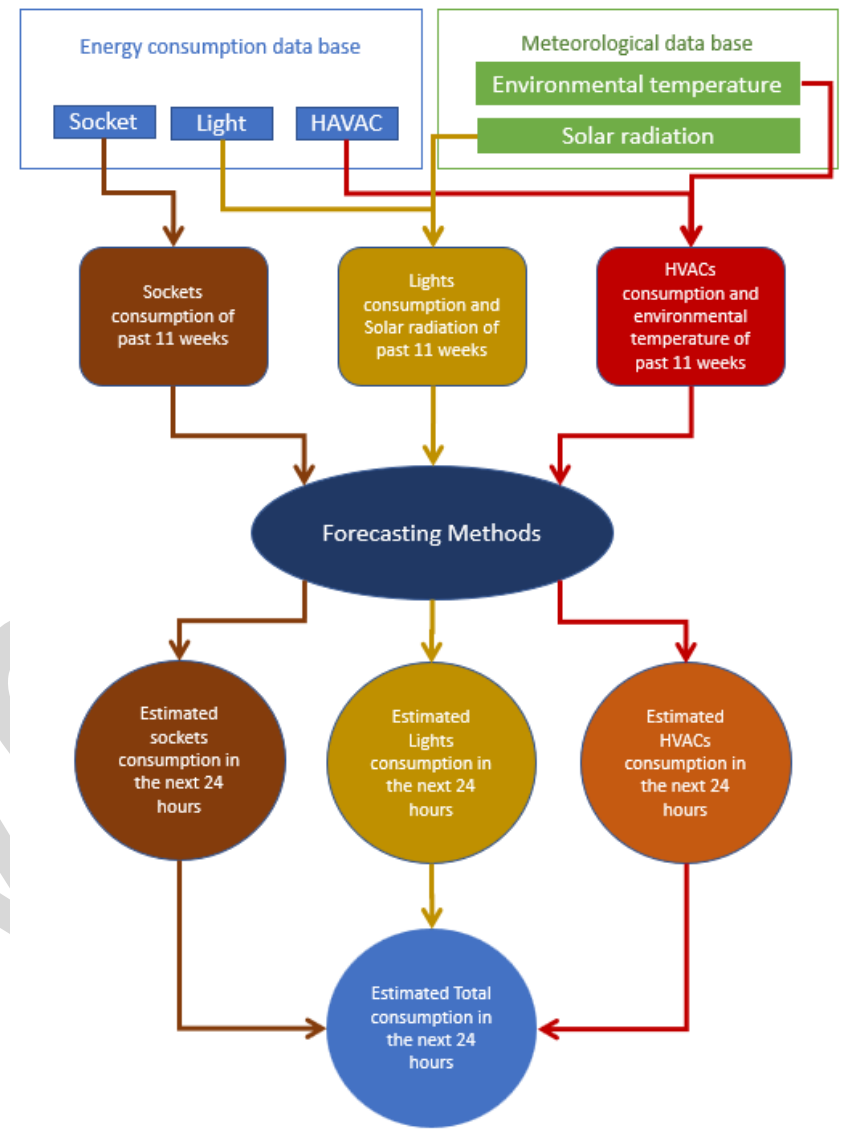

Figure 1 - The structure of the forecasting process in the second strategy

As the table 2 presents, the consumption values by the forecasting methods when the second strategy is used are closer to the real values. To evaluate the performance of the methods based on the second strategy figure 2 shows the MAPE errors of the forecasted values for 24 hours of 16/11/2018.

In the most hours of the day, the SVM presents a more honest perspective of the energy consumption, especially during the peak hours of the consumption in the office building namely from 9:00 to 18:00. However, in the case of some hours, it has a high error. Comparing the results of these two strategies shows that the methods are able to present a more trustable performance when a second variable is used in the training process. Figure 3 presents the average MAPE errors of the methods while the first and second strategy is used.

The average errors of the methods when the two proposed strategies have been used proves that in the case of the GFS.FR.MOGUL the average error is $10.01 \%$ for both strategies. It means that using a second variable such as solar radiation or environmental temperature does not influence the 
efficiency of this method. For HyFIS and WM can be concluded that these two forecasting methods have similar performance and can take significant advantage of having a second variable in the training process. The average error for HyFIS and WM when the first strategy is used is $18.84 \%$ and $18.79 \%$, while the second strategy is used, the presented errors of these methods are $13.50 \%$ and $13.16 \%$. The performance of the SVM also is improved when the metrological variables are used in the training process. However, as the SVM by the first strategy presents a low error, this improvement is not so high. The average error of SVM in the case of the first strategy is $9.44 \%$ and in the case of the second strategy is $9.11 \%$. The compression of these results shows that for both of the proposed strategies the SVM is able to present a more reliable day-ahead profile for energy consumption in an office building.

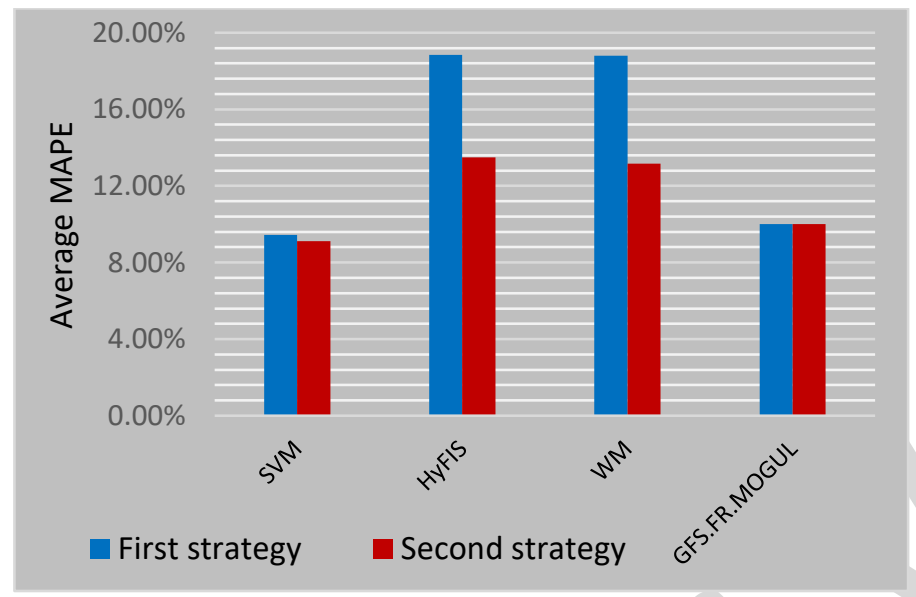

Figure 2 - Comparison of average MAPE errors

\section{CONCLUSIONS}

This work presents a forecasting approach which is able to estimate a day-ahead profile for energy consumption of an office building and presents a more trustable performance comparing to the previous approaches. Four forecasting methods namely Support Vector Machine (SVM), Hybrid Neural Fuzzy Interface System (HyFIS), Wang and Mendel's Fuzzy Rule Learning Method (WM) and a genetic fuzzy system for fuzzy rule learning based on the MOGUL methodology (GFS.FR.MOGUL) are proposed to be used in order to find the best forecasting model. Two training strategies have been used in this process to obtain the best results from the forecasting methods. In the first strategy, the consumption is divided into three values which correspond to the consumption of HVAC, lights and electrical sockets. The methods will be trained separately to predict the consumption of these three consumers in the next 24 hours, and the sum of these predicted values presents the final forecasted profile. The second strategy has a similar way of dividing the consumption as the first strategy but in the first strategy the methods are only trained by value of the energy consumption of the past 11 weeks, while in the second strategy in the case of HVACs the methods are trained by a combination on the energy consumption and environmental temperature. Also, to predict the consumption of the lights in the second strategy the methods are trained by the combination of the energy consumption and solar radiation of the related place.
The results of this study prove that in the case of most of the hours using the metrological variables can help the methods to predict more trustable results. The SVM, HyFIS, and WM present a lower average error when the second strategy is used, however in the case of SVM; the difference is small because this method even in the case of the first strategy presents a low error. About GFS.FR.MOGUL can conclude that since this method has the same average error when these two strategies are used, the usage of a second variable does not affect the performance of this method. Between these forecasting methods, the SVM in the case of both strategies presents a better profile for the energy consumption and when the second strategy is used to train this method the most reliable results are achieved.

As future work, the influence of the other metrological variables such as humidity and internal temperature is considered as well as using multiple learning form the results of different forecasting methods.

Table 2 - Forecasted consumption values based on the second strategy

Hour Real SVM HyFIS WM GFS.FR.MOGUL

\begin{tabular}{l|lllll}
\hline $0: 00$ & 1502 & 1580 & 1716 & 1716 & 1683 \\
$1: 00$ & 1602 & 1577 & 1350 & 1350 & 1785 \\
$2: 00$ & 1462 & 1535 & 1347 & 1347 & 1728 \\
$3: 00$ & 1499 & 1706 & 1833 & 1830 & 1752 \\
$4: 00$ & 1467 & 1568 & 1836 & 1836 & 1572 \\
$5: 00$ & 1525 & 1529 & 1557 & 1552 & 1769 \\
$6: 00$ & 2065 & 1548 & 1659 & 1660 & 1555 \\
$7: 00$ & 1447 & 1509 & 1376 & 1377 & 1807 \\
$8: 00$ & 1534 & 1528 & 1438 & 1437 & 1598 \\
$9: 00$ & 2186 & 2140 & 1638 & 1658 & 2360 \\
$10: 00$ & 2447 & 2510 & 2517 & 2454 & 2505 \\
$11: 00$ & 2640 & 3233 & 3357 & 3357 & 2834 \\
$12: 00$ & 3235 & 2952 & 2930 & 2930 & 3184 \\
$13: 00$ & 3566 & 3302 & 3617 & 3589 & 3232 \\
$14: 00$ & 3164 & 3823 & 3646 & 3620 & 3556 \\
$15: 00$ & 3621 & 3652 & 4119 & 4126 & 3880 \\
$16: 00$ & 3710 & 3834 & 3165 & 3208 & 3722 \\
$17: 00$ & 3399 & 3602 & 3859 & 3785 & 3585 \\
$18: 00$ & 3031 & 2557 & 2536 & 2521 & 2702 \\
$19: 00$ & 2324 & 1888 & 2107 & 2110 & 2140 \\
$20: 00$ & 1791 & 1708 & 2072 & 2072 & 1854 \\
$21: 00$ & 2388 & 1674 & 1705 & 1709 & 2022 \\
$22: 00$ & 1820 & 1616 & 1817 & 1832 & 1997 \\
$23: 00$ & 1756 & 1786 & 1537 & 1538 & 1814 \\
\hline & & & & & \\
\hline
\end{tabular}




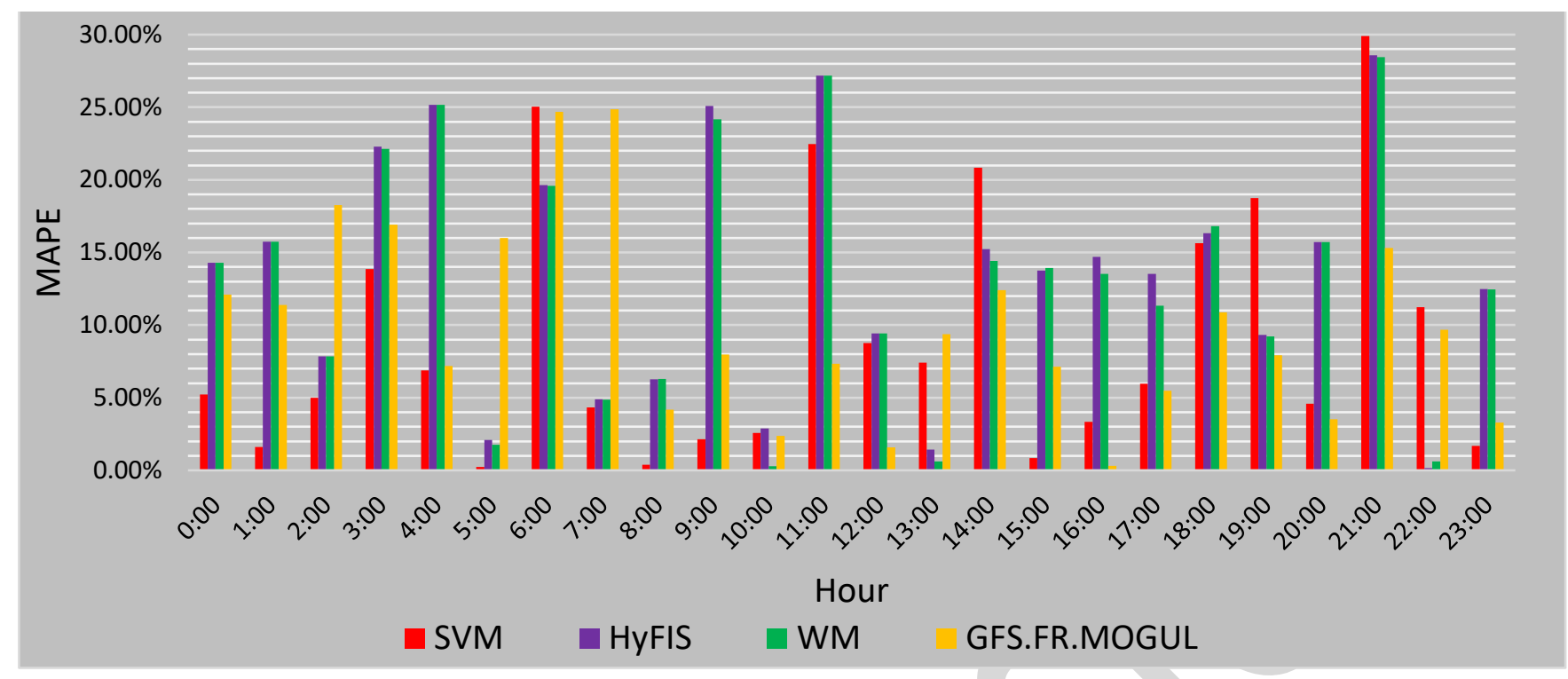

Figure 3 - MAPE errors of the estimated values based on the second strategy

\section{REFERENCES}

[1] European Commission, "2030 Energy Strategy," 2017.

[2] Z. Geng, A. J. Conejo, Q. Chen, Q. Xia, and C. Kang, "Electricity production scheduling under uncertainty: Max social welfare vs. min emission vs. max renewable production," Appl. Energy, vol. 193, pp. 540-549, 2017.

[3] J. Soares, T. Pinto, F. Lezama, and H. Morais, "Survey on Complex Optimization and Simulation for the New Power Systems Paradigm," Complexity, vol. 2018, pp. 1-32, Aug. 2018.

[4] I. Okumus and A. Dinler, "Current status of wind energy forecasting and a hybrid method for hourly predictions," Energy Convers. Manag., vol. 123, pp. 362-371, 2016

[5] A. Gazafroudi, F. Prieto-Castrillo, T. Pinto, J. Prieto, J. Corchado, and J. Bajo, "Energy Flexibility Management Based on Predictive Dispatch Model of Domestic Energy Management System," Energies, vol. 10, no. 9, p. 1397, Sep. 2017.

[6] M. A. Mat Daut, M. Y. Hassan, H. Abdullah, H. A. Rahman, M. P. Abdullah, and F. Hussin, "Building electrical energy consumption forecasting analysis using conventional and artificial intelligence methods: A review," Renew. Sustain. Energy Rev., vol. 70, pp. 1108$1118,2017$.

[7] J. M. Corrêa, A. C. Neto, L. A. Teixeira Júnior, E. M. C. Franco, and A. E. Faria, "Time series forecasting with the WARIMAX-GARCH method," Neurocomputing, vol. 216, pp. 805-815, 2016.

[8] S. Torbat, M. Khashei, and M. Bijari, "A hybrid probabilistic fuzzy ARIMA model for consumption forecasting in commodity markets," Econ. Anal. Policy, vol. 58, pp. 22-31, 2018.

[9] M. Q. Raza and A. Khosravi, "A review on artificial intelligence based load demand forecasting techniques for smart grid and buildings," Renew. Sustain. Energy Rev., vol. 50, pp. 1352-1372, 2015.

[10] Y.-R. Zeng, Y. Zeng, B. Choi, and L. Wang, "Multifactor-influenced energy consumption forecasting using enhanced back-propagation neural network," Energy, vol. 127, pp. 381-396, 2017.

[11] V. H. Hinojosa and A. Hoese, "Short-term load forecasting using fuzzy inductive reasoning and evolutionary algorithms," IEEE Trans. Power Syst., vol. 25, no. 1, pp. 565-574, 2010.

[12] A. Azadeh, S. M. Asadzadeh, G. H. Mirseraji, and M. Saberi, “An emotional learning-neuro-fuzzy inference approach for optimum training and forecasting of gas consumption estimation models with cognitive data," Technol. Forecast. Soc. Change, vol. 91, pp. 47-63, 2015.

[13] M. S. Al-Musaylh, R. C. Deo, J. F. Adamowski, and Y. Li, "Short-term electricity demand forecasting with MARS, SVR and ARIMA models using aggregated demand data in Queensland, Australia," Adv. Eng. Informatics, vol. 35, pp. 1-16, 2018.

[14] X. Zhang, J, Wang, and K. Zhang, "Short-term electric load forecasting based on singular spectrum analysis and support vector machine optimized by Cuckoo search algorithm," Electr. Power Syst. Res., vol. 146, pp. 270-285, 2017.

[15]A. Jozi, T. Pinto, I. Praca, F. Silva, B. Teixeira, and Z. Vale, "Energy consumption forecasting based on Hybrid Neural Fuzzy Inference System," in 2016 IEEE Symposium Series on Computational Intelligence (SSCI), 2016, pp. 1-5.

[16] A. Jozi, T. Pinto, I. Praça, F. Silva, B. Teixeira, and Z. Vale, "Wang and Mendel's Fuzzy Rule Learning Method for Energy Consumption Forecasting considering the Influence of Environmental Temperature," in Giis 2016, 2016.

[17] A. Jozi, T. Pinto, I. Praça, and Z. Vale, "Day ahead electricity consumption forecasting with MOGUL learning model," in IEEE World Congress on Computational Intelligence, 2018.

[18] L. Gomes, P. Faria, H. Morais, Z. Vale, and C. Ramos, "Distributed, agentbased intelligent system for demand response program simulation in smart grids," IEEE Intell. Syst., vol. 29, no. 1, pp. 56-65, 2014.

[19] A. L. V. Vapnik, "Pattern recognition using generalized portrait method," Autom. Remote Control, vol. 24, pp. 774-780, 1963.

[20]B. E. Boser, I. M. Guyon, and V. N. Vapnik, "A training algorithm for optimal margin classifiers," Proc. fifth Annu. Work. Comput. Learn. theory - COLT'92, pp. 144-152, 1992.

[21] D. Meyer et al., "Misc functions of the department of statistics, probability theory group," 2015.

[22] L. S. Riza, C. Bergmeir, F. Herrera, and J. M. Benítez, “ $\{$ frbs $\}$ : Fuzzy RuleBased Systems for Classification and Regression in $\{\mathrm{R}\}, " R$ Packag. version 3.1-0, 2015

[23] L. A. Zadeh, "Fuzzy sets," Inf. Control, vol. 8, no. 3, pp. 338-353, Jun. 1965.

[24] J. Kim and N. Kasabov, "HyFIS: adaptive neuro-fuzzy inference systems and their application to nonlinear dynamical systems," Neural Networks, vol. 12, no. 9, pp. 1301-1319, 1999.

[25] W. Pedrycz, P. Ekel, and R. Parreiras, "Notions and Concepts of Fuzzy Sets: An Introduction,” Fuzzy Multicriteria Decis., pp. 21-62, 2010. 


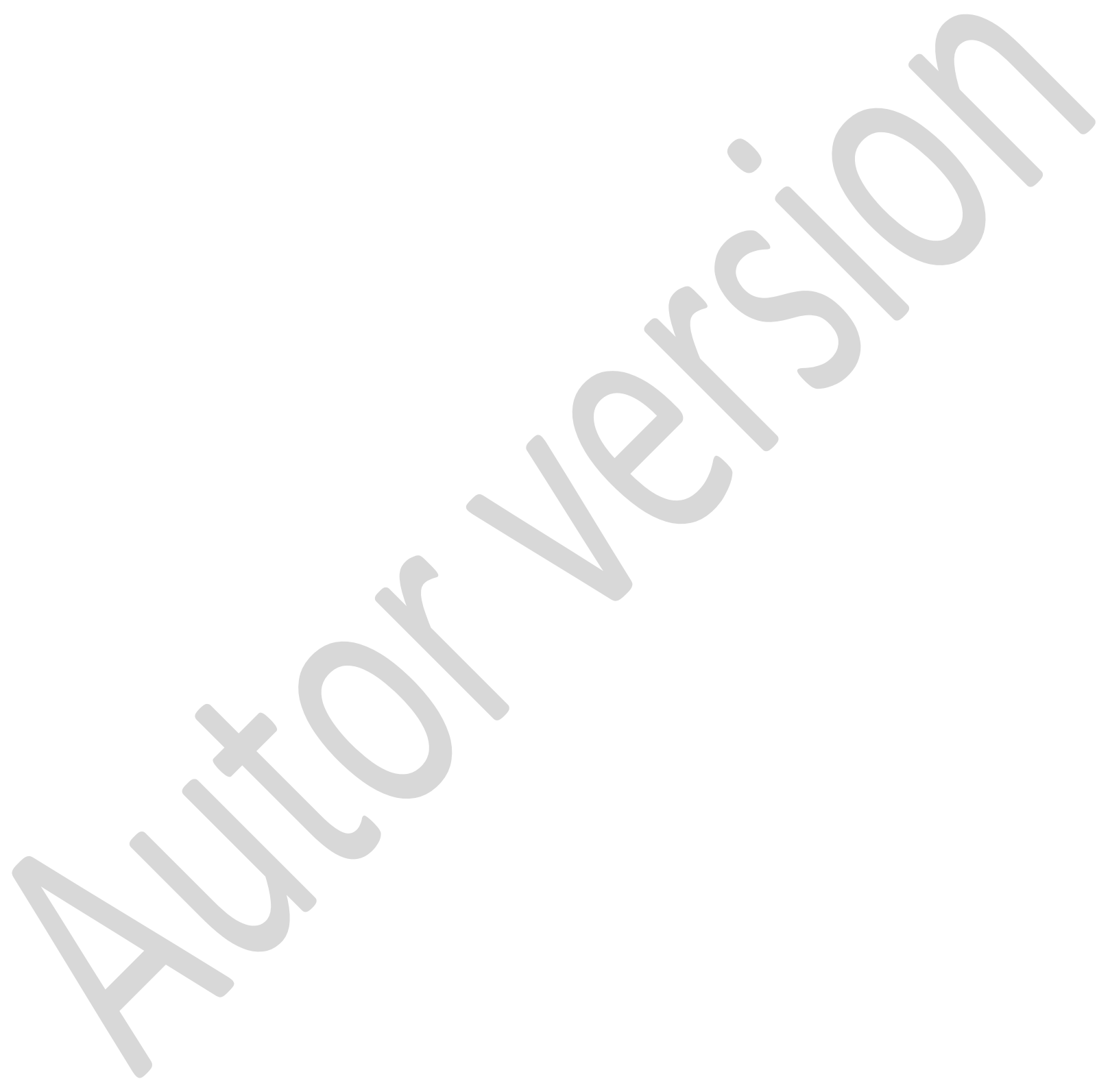

